\title{
First JAM results on the determination of polarized parton distributions
}

\author{
A. Accardi* \\ Hampton U. and Jefferson Lab \\ E-mail: accardiejlab.org \\ P. Jimenez-Delgado \\ Jefferson Lab \\ E-mail: pedro@jlab.org \\ W. Melnitchouk \\ Jefferson Lab \\ E-mail: wmelnitcejlab.org
}

The Jefferson Lab Angular Momentum (JAM) Collaboration is a new initiative to study the angular momentum dependent structure of the nucleon. First results on the determination of spindependent parton distribution functions at intermediate and large $x$ from world data on polarized deep-inelastic scattering are presented. Different aspects of global QCD analysis are discussed, including the effects of nuclear structure of deuterium and ${ }^{3} \mathrm{He}$ targets, target mass corrections and higher twist contributions to the $g_{1}$ and $g_{2}$ structure functions.

XXI International Workshop on Deep-Inelastic Scattering and Related Subject - DIS2013, 22-26 April, 2013

Marseilles, France

\footnotetext{
* Speaker.
} 
Introduction. The JAM (Jefferson Lab Angular Momentum) Collaboration [1] is a new enterprise involving theorists and experimentalists from the nuclear and hadron physics community to study the quark and gluon spin structure of the nucleon through global fits of spin-dependent parton distribution functions (PDFs). In parallel with the fitting effort, an interactive, public database containing all available data on polarized scattering experiments is being developed.

The initial goal is to perform a global next-to-leading order (NLO) analysis of spin dependent DIS structure functions and asymmetries, paying particular attention to the high- $x$ and low- $Q^{2}$ regions. The importance of obtaining reliable parton distribution fits at large $x$ is manyfold [2]. Accurate PDFs at large $x$ are necessary to reduce the uncertainty on QCD backgrounds in searches for new physics in high- $p_{T}$ spectra, or for new heavy particles at the LHC. At the other end of the spectrum, the behavior of quarks at $x \rightarrow 1$ reflects the nonperturbative structure of the nucleon, with PDF ratios such as $d / u, \Delta u / u$ or $\Delta d / d$ sensitive to the inter-quark dynamics at long distances $[3,4]$. Finally, the uncertainty in the extraction of the gluon helicity PDF at small $x$, which can be obtained from forward particle production in polarized $p p$ collisions, is fundamentally limited by the uncertainty on the large- $x$ PDF of the quark against which the gluon scatters.

Including large- $x$ and low- $Q^{2}$ data in a global fit requires careful consideration of various finite$Q^{2}$ corrections to perturbative calculations, such as those arising from target mass and higher twist effects, as well as nuclear corrections associated with the use of Deuteron and ${ }^{3} \mathrm{He}$ targets (which are necessary for $u$ and $d$ quark flavor separation). These have been shown in recent unpolarized fits to be essential to correctly describe DIS data at low invariant mass $W$ [5-8] and are even more important in polarized fits, where low- $W$ DIS data comprise a substantial fraction of the available data.

To study the impact of large- $x$ and low- $Q^{2}$ effects in polarized fits, we begin with a baseline fit which makes use of inclusive DIS data [9]. The novelties of the JAM fit framework are discussed below, and a table comparing the JAM framework with that of other global analysis groups is available in this talk's slides [10].

Theoretical framework. The JAM fits presented here include two kinds of $\mathscr{O}\left(1 / Q^{2}\right)$ corrections: target mass corrections (TMCs) and higher twist (HT) contributions. TMCs are treated in the OPE formalism [11], while higher twist terms are fitted to data. In contrast to other global fits, we include a complete treatment of HT corrections for both the $g_{1}$ and $g_{2}$ structure functions within the same analysis. This has previously been investigated in by Blümlein and Böttcher separately for $g_{1}$ and $g_{2}[12,13]$, and is carried out here in the context of a simultaneous global PDF fit to both $g_{1}$ and $g_{2}$ data.

To account for the nuclear corrections, previous polarized global analyses have relied exclusively on the effective polarization ansatz, in which the polarized PDF in the nucleus, $\Delta f_{i}^{A}$, is related to the polarized PDFs in the proton and neutron by $\Delta f_{i}^{A} \approx\langle\sigma\rangle^{p} \Delta f_{i}^{p}+\langle\sigma\rangle^{n} \Delta f_{i}^{n}$, with $\langle\sigma\rangle^{p(n)}$ the average polarization of the proton (neutron) in the nucleus. The JAM analysis is the first attempt to systematically incorporate the effects of nuclear binding and Fermi motion in deuterium and ${ }^{3} \mathrm{He}[14,15]$. Corrections due to the struck nucleon being off-shell will be included in subsequent analyses, but are expected to play a less important role than for unpolarized PDFs [5, 6] because of the generally larger uncertainties on the polarized DIS data at high $x$. 
The last important methodological aspect of the JAM fits is the use of the measured parallel and perpendicular $A_{\perp}$ asymmetries, rather than reconstructed structure functions, and the calculation of $F_{1}$ from an unpolarized fit using a common set of theoretical corrections [8]. This allows one to benefit from the largest possible unpolarized data set covering the same kinematics, and avoids attributing to polarized PDFs effects neglected in the unpolarized fits.

Fitting strategy. The DIS data set [1] considered in the fits presented here include polarized DIS measurements from SLAC, SMC, COMPASS, HERMES and Jefferson Lab on proton, deuterium and ${ }^{3} \mathrm{He}$ targets [10]. Perturbative calculations are performed in Mellin moment space. The leading twist contribution $g_{1}^{(\tau=2)}$ is calculated at NLO, and for $g_{2}^{(\tau=2)}$ we use the Wandzura-Wilczek relation. Higher twist contributions to proton and neutron structure functions are included according to [11]

$$
\begin{aligned}
& g_{1}=g_{1}^{(\tau=2)}+g_{1}^{(\tau=3)}+g_{1}^{(\tau=4)}, \\
& g_{2}=g_{2}^{(\tau=2)}+g_{2}^{(\tau=3)},
\end{aligned}
$$

where the twist- 3 contribution to $g_{1}$ is determined by the twist-3 part of $g_{2}$,

$$
g_{1}^{(\tau=3)}\left(x, Q^{2}\right)=4 x^{2} \frac{M^{2}}{Q^{2}}\left(g_{2}^{(\tau=3)}\left(x, Q^{2}\right)-2 \int_{x}^{1} \frac{d y}{y} g_{2}^{(\tau=3)}\left(x, Q^{2}\right)\right) .
$$

The function $g_{2}^{(\tau=3)}$ is parametrized following Braun et al. [16], and a spline approximation is used for $g_{1}^{(\tau=4)}\left(x, Q^{2}\right)=h(x) / Q^{2}$. The QCD evolution of $h$ and $g_{2}^{(\tau=3)}$ is for now neglected compared to the precision of the experimental data.

For the PDF parametrization at the initial scale $\mu_{0}=1 \mathrm{GeV}^{2}$, we use the form

$$
x \Delta q^{+}\left(x, \mu_{0}\right) \equiv x(\Delta q+\Delta \bar{q})=N_{q} x^{a_{q}}(1-x)^{b_{q}}\left(1+B_{q} x\right),
$$

for $q=u, d$. For the sea quarks, the DIS data alone cannot constrain the strange quark PDF $\Delta s$, so a fixed shape determined by counting rules at large $x$, and reproducing the behavior of the $u$ and $d$ quark PDFs at small $x$, was adopted. The normalizations of the quark distributions was determined from weak hyperon decays. Although $\Delta g$ formally contributes to DIS through QCD evolution, in practice the data give only very mild constraints. Therefore, a fixed shape in $x$, comparable with that from the DSSV09 fit [17].

Results. In Fig. 1, we sequentially study the effect of the various theoretical corrections on the $\Delta u^{+}$and $\Delta d^{+}$distributions starting from a fit with no TMC and HT corrections and using the effective polarization approximation without smearing for nuclear targets. At small values of $x$ the effects of the theoretical corrections is negligible; however, at $x \gtrsim 0.3$ they can give up to $20 \%$ corrections for $\Delta u^{+}$and more than $50 \%$ corrections for $\Delta d^{+}$(the relative correction can be even larger at $x \gtrsim 0.8$, although the PDFs are not constrained in this region).

The same effects are more clearly illustrated through the polarization ratios $\Delta u^{+} / u^{+}$and $\Delta d^{+} / d^{+}$, where the unpolarized distributions are fitted within the same theoretical framework as the polarized [8], thereby eliminating bias arising from assumptions about spin-averaged PDF input. In the intermediate- $x$ region the ratios for both $u$ and $d$ quarks are generally consistent with the 

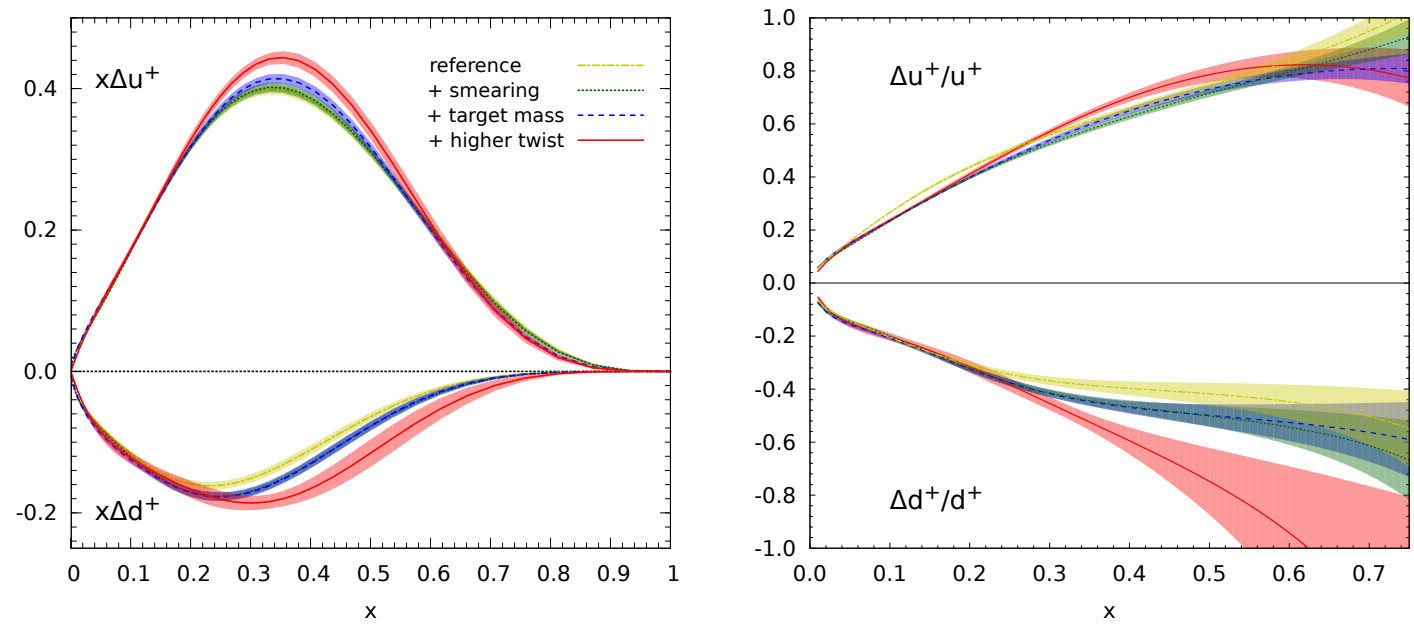

Figure 1: (Left) Polarized $x \Delta u^{+}$and $x \Delta d^{+}$distributions and their uncertainties for the JAM13 PDF set at $Q^{2}=1 \mathrm{GeV}^{2}$, illustrating the effects of the nuclear smearing (green dotted), target mass (blue dashed) and higher twist (red solid) corrections, relative to the reference fit (yellow dot-dashed). (Right) Corresponding ratios of polarized to unpolarized distributions.

symmetric quark model expectations, with the $\Delta u^{+} / u^{+}$increasing towards unity at larger $x$. The $\Delta d^{+} / d^{+}$ratio, on the other hand, remains negative for all $x$ where it is constrained, and shows no indication of the upturn predicted by the helicity conservation models $[18,19]$. The nuclear and finite- $Q^{2}$ effects can significantly impact the limiting behavior as $x \rightarrow 1$, and clearly additional data are needed to constrain their $x$ dependence at high $x$.

The extracted HT terms are presented in Fig. 2, which clearly demonstrates that it is possible to simultaneously extract the HT contributions to both $g_{1}$ and $g_{2}$ from the present data. The $g_{1}^{(\tau=3)}$ term is clearly non-negligible, and impacts the extraction of the twist- 4 term at $x \gtrsim 0.3$. For the proton, we observe qualitative agreement with $g_{1}^{(\tau=4)}$ extracted by Leader et al. [20], and with $g_{2}^{(\tau=3)}$ obtained by Accardi et al. [21] and Blümlein and Böttcher [13]. For the neutron the results differ from those of Leader et al. [20] for $g_{1}^{(\tau=4)}$. This may be due to the nuclear smearing corrections considered in the JAM fits, which substantially modify the extracted $\Delta d^{+}$quark distribution. The neutron contribution to $g_{2}^{(\tau=3)}$ is largely compatible with zero, in contrast to Ref. [21]. The Jefferson Lab E99-117 data are found to have a large impact on the neutron's $g_{1}$ fit, and practically determine its twist- 4 contribution.

Conclusions. The first results from the JAM Collaboration on polarized PDF fits demonstrate the importance of an accurate treatment of nuclear corrections taking into account the nuclear smearing effects neglected in the more common treatments in terms of effective polarizations. These corrections have the largest impact on the $\Delta d^{+}$distribution. Furthermore, after subtraction of target mass corrections, a simultaneous fit of the higher twist contributions to the $g_{1}$ and $g_{2}$ structure functions is possible, and indeed necessary.

Work in progress includes a detailed study of the impact of more recent Jefferson Lab data on the leading twist and higher twist distributions at high $x$. The longer term plans include the analysis of semi-inclusive DIS data and polarized proton-proton asymmetries from RHIC. 

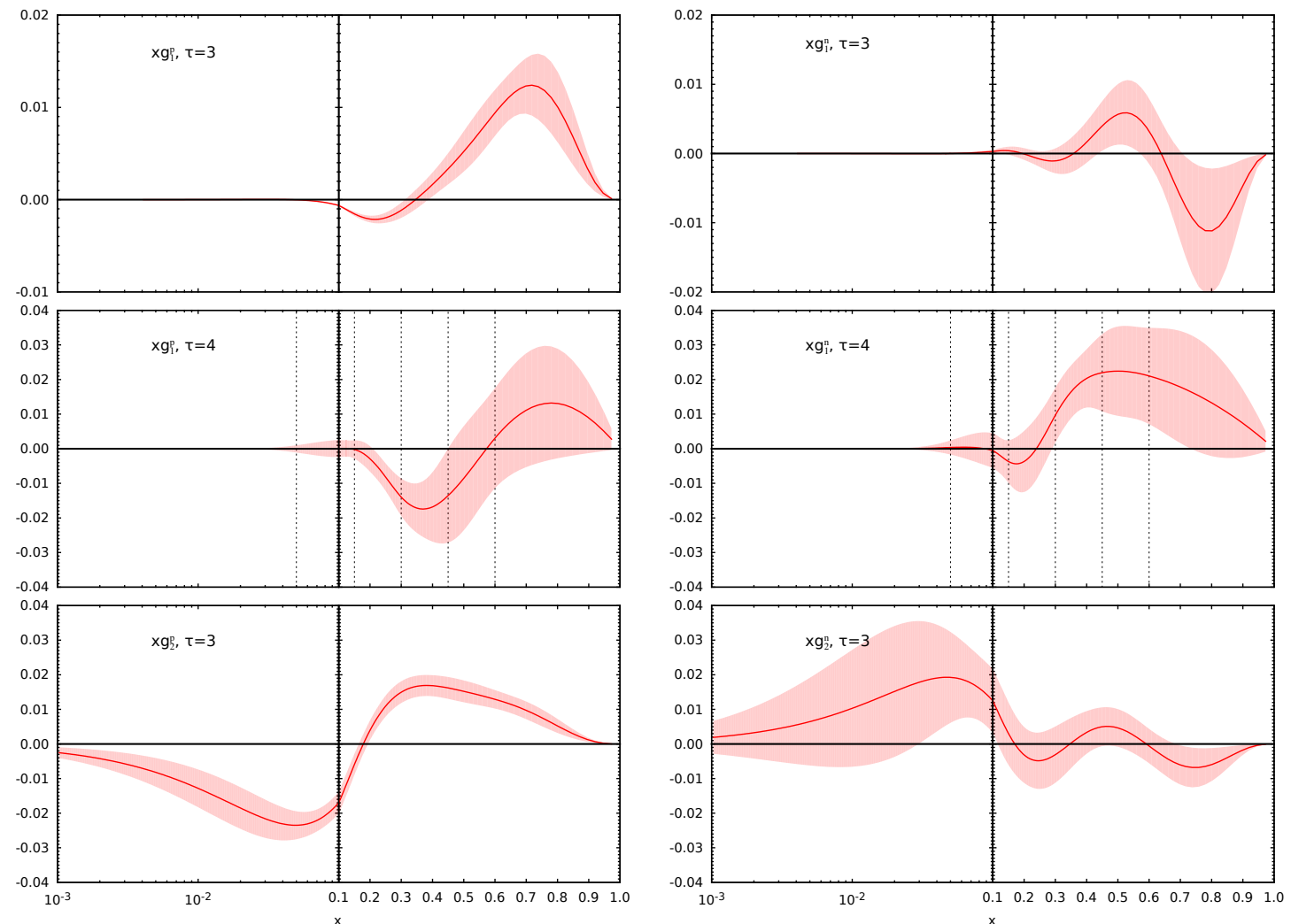

Figure 2: The proton ((left) and neutron (right) higher twist terms determined in the JAM fit.

Acknowledgments: This work was supported by the DOE contract No. DE-AC05-06OR23177, under which Jefferson Science Associates, LLC operates Jefferson Lab, and by the DOE contract DE-SC008791.

\section{References}

[1] The Jefferson Lab Angular Momentum (JAM) collaboration website, http: / /www.jlab.org/jam.

[2] A. Accardi, "PDF uncertainties at large $x$ and gauge boson production," PoS ConfinementX (2012) 227.

[3] W. Melnitchouk and A. W. Thomas, Phys. Lett. B 377 (1996) 11.

[4] P. Jimenez-Delgado, W. Melnitchouk and J. F. Owens, "Parton momentum and helicity distributions in the nucleon," to appear in J. Phys. G (2013), arXiv:1306.6515 [hep-ph].

[5] J. F. Owens, A. Accardi and W. Melnitchouk, "Global parton distributions with nuclear and finite- $Q^{2}$ corrections,” Phys. Rev. D 87 (2013) 094012.

[6] A. D. Martin et al., "Extended parameterisations for MSTW PDFs and their effect on lepton charge asymmetry from $W$ decays,” Eur. Phys. J. C 73 (2013) 2318.

[7] R. D. Ball et al., "Unbiased determination of polarized parton distributions and their uncertainties," Nucl. Phys. B874 (2013) 36 [arXiv:1303.7236 [hep-ph]]. 
[8] P. Jimenez Delgado and E. Reya, in preparation.

[9] P. Jimenez-Delgado, A. Accardi and W. Melnitchouk, in preparation.

[10] Slides of this talk, http://dis2013.in2p3.fr/.

[11] J. Blümlein and A. Tkabladze, "Target mass corrections for polarized structure functions and new sum rules,” Nucl. Phys. B553 (1999) 427.

[12] J. Blümlein and H. Böttcher, “QCD Analysis of Polarized Deep Inelastic Scattering Data," Nucl. Phys. B841 (2010) 205.

[13] J. Blümlein and H. Böttcher, "Higher Twist contributions to the structure functions $F_{2}\left(x, Q^{2}\right)$ and $g_{2}\left(x, Q^{2}\right), "$ arXiv:1207.3170 [hep-ph].

[14] S. A. Kulagin and W. Melnitchouk, Phys. Rev. C 77 (2008) 015210.

[15] S. A. Kulagin and W. Melnitchouk, Phys. Rev. C 78 (2008) 065203.

[16] V. M. Braun, T. Lautenschlager, A. N. Manashov and B. Pirnay, "Higher twist parton distributions from light-cone wave functions,” Phys. Rev. D 83 (2011) 094023.

[17] D. de Florian, R. Sassot, M. Stratmann and W. Vogelsang, Phys. Rev. D 80 (2009) 034030.

[18] R. Blankenbecler and S. J. Brodsky, Phys. Rev. D 10 (1974) 2973.

[19] G. R. Farrar and D. R. Jackson, Phys. Rev. Lett. 35 (1975) 1416.

[20] E. Leader, A. V. Sidorov and D. B. Stamenov, "Determination of polarized PDFs from a QCD analysis of inclusive and semi-inclusive deep-inelastic scattering data," Phys. Rev. D 82 (2010) 114018.

[21] A. Accardi, A. Bacchetta, W. Melnitchouk and M. Schlegel, "What can break the Wandzura-Wilczek relation?," JHEP 0911 (2009) 093. 